\title{
CICLO DE CORTE ECONÔMICO ÓTIMO EM FLORESTA OMBRÓFILA DENSA DE TERRA FIRME SOB MANEJO FLORESTAL SUSTENTÁVEL, AMAZÔNIA ORIENTAL ${ }^{1}$
}

\author{
Deoclides Ricardo de Souza², Agostinho Lopes de Souza ${ }^{3}$, Márcio Lopes da Silva² e Flávio Lopes \\ Rodrigues ${ }^{4}$
}

\begin{abstract}
RESUMO - Este estudo teve como objetivo estimar o ciclo de corte e o estoque de colheita ótimo, no qual a taxa anual do crescimento da floresta se iguala à taxa anual de juros oferecida pelo mercado de capital. A pesquisa foi conduzida na Unidade de Manejo Florestal (UMF) da Fazenda Tracajás (02³5'53'’S e 4747'10’'W), empresa Nova Era Agroflorestal, município de Paragominas, Estado do Pará, Brasil. A unidade de manejo florestal foi estratificada em classes I, II e III de estoques volumétricos, empregando-se análises de agrupamento e discriminante. Em cada classe de estoque foram instaladas aleatoriamente cinco parcelas de 100 x $100 \mathrm{~m}$ (1,0 ha), para medição dos indivíduos com dap $\geq 15 \mathrm{~cm}$. No centro de cada parcela foi instalada uma subparcela de 10 x $100 \mathrm{~m}(0,1 \mathrm{ha})$, para medição dos indivíduos com $5 \mathrm{~cm} \leq$ dap $<15 \mathrm{~cm}$. Na classe I de estoque, os ciclos econômicos ótimos foram de 13, 12 e 8 anos; na classe II, de 18, 12 e 12 anos; e na classe III, de 22, 12 e 14 anos, a um incremento médio anual de 3,0 $\mathrm{m}^{3} / \mathrm{ha} / \mathrm{ano}$. Verificaram-se maiores taxas de remuneração do capital investido no manejo nos menores ciclos de corte, independentemente dos níveis de colheita do estoque comercial. Maiores incrementos anuais em volume resultaram em maiores taxas de valoração da floresta. Maiores volumes colhidos implicaram ciclos de corte mais longos numa mesma taxa de crescimento da floresta. Contudo, dentro de certos limites, maiores volumes colhidos podem resultar biologicamente em maiores taxas de crescimento do estoque remanescente. Isso ocorre, sobretudo, quando se aplicam tratamentos silviculturais.
\end{abstract}

Palavras-chave: Floresta tropical, classe de estoque, ciclo de corte, análise econômica.

\section{OPTIMUM ECONOMIC CUTTING CYCLE IN A TERRA FIRME DENSE OMBROPHYLOUS FOREST UNDER SUSTAINED MANAGEMENT, EASTERN AMAZON}

\begin{abstract}
The objective of the present study was to estimate the optimum cutting cycle and harvest stock, where forest annual growing rate equals the annual interest rate offered by the financial market. The research

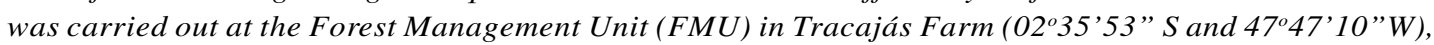
owned by Nova Era Agroflorestal, in Paragominas, Pará, Brazil. The forest was stratified in three homogeneous areas denominated classes of volumetric stock I, II and III, through multivariate analysis (cluster and discriminant analysis). In each of the stock classes, five $100 \times 100 \mathrm{~m}$ plots (1 ha) were randomly set up to measure individuals with $\mathrm{dbh} \geq 15 \mathrm{~cm}$. In the center of each $100 \times 100 \mathrm{~m}$ plot, a $10 \times 100 \mathrm{~m}$ subplot $(0,1 \mathrm{ha})$ was installed to measure individuals with $5 \mathrm{~cm} \leq \mathrm{dbh}<15 \mathrm{~cm}$. In stock class I, the optimum economic cycles were 13, 12,
\end{abstract}

${ }^{1}$ Recebido para publicação em 21.10.2003 e aceito para publicação em 10.8.2004.

${ }^{2}$ Departamento de Engenharia Agronômica da Universidade Federal de Sergipe - UFS, São Cristovão, SE, CEP 49100-000. <souzadr@hotmail.com>.

${ }^{3}$ Departamento de Engenharia Florestal da Universidade Federal de Viçosa. <alsouza@ mail.ufv.br>.

${ }^{4}$ Centro de Ciências Agrárias - Universidade Federal do Espírito Santo, Alegre, Espírito Santo. 
and 8 years; in stock class II, 18, 12 and 12 years; in stock class III, 22, 12 and 14 years, considering an average annual increment of $3,0 \mathrm{~m}^{3} / \mathrm{ha}$ year. Higher remuneration rates for the invested capital were verified for the shorter cutting cycles, regardless of the commercial harvest. Higher annual volume increments resulted in a higher valorization of the forest. Higher harvested volumes implicated in longer cutting cycles for a same forest growing rate. However, within certain limits, higher harvested volumes can biologically result in higher growing rates of the remaining stock. This occurs mostly when silvicultural treatments are applied.

Key words: Tropical forest, stock classes, cutting cycle, economic analysis.

\section{INTRODUÇÃO}

A complexibilidade dos ecossistemas de florestas tropicais naturais dificulta as avaliações de parâmetros biológicos, principalmente os relacionados com o crescimento e a produção. Pelo fato de essas florestas não estarem atualmente estruturadas para a produção sustentável, é necessária a fixação do tamanho ótimo de ciclos de corte para atingir uma distribuição diamétrica e uma composição de espécies adequadas aos propósitos do manejo, de modo a permitir a regeneração, o crescimento e o desenvolvimento prioritariamente de árvores de valor comercial.

As áreas de florestas naturais sob manejo são colhidas segundo uma seqüência estabelecida. O tempo que a floresta leva para recompor o nível de estoque de colheita é denominado ciclo de corte. Por conseguinte, o ciclo de corte está relacionado com a estrutura da floresta remanescente, as características ecofisiológicas das espécies, as taxas de crescimento e regeneração das espécies e a intensidade de manejo praticado (SOUZA, 1999). Assim, a determinação do ciclo de corte consiste na otimização dos fatores biológicos e econômicos que propiciam o maior retorno líquido do capital investido na atividade manejo. Portanto, o conhecimento do ciclo de corte é importante, uma vez que influencia tanto a recuperação e a formação do material lenhoso da floresta quanto a prognose da produção e avaliação dos retornos financeiros e econômicos provenientes da madeira colhida (CHICHORRO, 2000). Assim, o ciclo de corte atua como instrumento regulador da quantidade de madeira removida, controlando o nível de estoque de crescimento.

Outra questão fundamental na determinação do ciclo de corte está relacionada à taxa de juros aplicada na avaliação econômica do manejo florestal; altas taxas de desconto usadas em análises financeiras inviabilizam o manejo das florestas tropicais naturais.
O emprego do recurso florestal com taxas de juros compatíveis com a taxa de crescimento da floresta garante os retornos do investimento na atividade de manejo, bem como a utilização do recurso sem comprometer a sua capacidade de renovação e sustentação. Contudo, estudos sobre a determinação do ciclo de corte em florestas tropicais naturais (ARAUJO et al., 1993; BOM, 1996; SILVA, 1996; SOUZA, 1999) são ainda insuficientes, devido à escassez de dados de crescimento e produção, de critérios econômicos em termos de taxas de investimentos e de valoração dos diferentes produtos florestais, bens e serviços ecológicos. Este estudo teve como objetivo estimar o ciclo de corte e o estoque de colheita ótimo, no qual a taxa anual do crescimento da floresta se iguala à taxa anual de juros oferecida pelo mercado de capital.

\section{REVISÃO BIBLIOGRÁFICA}

\subsection{Incremento}

As diferenças entre os tipos florestais dificultam a comparação de taxas de crescimento e produção. Estudos feitos por Silva et al. (1995, 1996 e 1999) em florestas tropicais naturais, no Estado do Pará, constataram que a produção líquida volumétrica variou de 1,6 $\mathrm{m}^{3} / \mathrm{ha}$ /ano na floresta primária não explorada a $4,8 \mathrm{~m}^{3} /$ ha/ano na floresta explorada sem tratamento silvicultural. Na floresta secundária, a produção volumétrica foi de $3,5 \mathrm{~m}^{3} / \mathrm{ha} / \mathrm{ano}$, e os incrementos anuais em volume das espécies comerciais variaram entre 1,0 ; 1,5 ; e $1,8 \mathrm{~m}^{3} / \mathrm{ha}$ /ano.

Em floresta atlântica, no Estado de Minas Gerais, Ferreira (1997) constatou, ao final de 10 anos de monitoramento em um experimento de manejo de mata secundária em que foram aplicados cortes seletivos, incrementos anuais em volume entre 3,9 e $3,7 \mathrm{~m}^{3} / \mathrm{ha} /$ ano. 
Alder e Silva (2000) observaram incremento anual em volume de $2,56 \mathrm{~m}^{3} / \mathrm{ha} /$ ano nas espécies com dap $\geq$ $45 \mathrm{~cm}$, após 17 anos de monitoramento em uma floresta equatorial de terra firme, na Amazônia.

Maitre (1991), analisando três áreas de floresta tropical na Costa do Marfim, observou incrementos anuais em volume entre 0,7 e $1,8 \mathrm{~m}^{3} / \mathrm{ha} /$ ano na área não-explorada, $2,5 \mathrm{~m}^{3} / \mathrm{ha} /$ ano na área explorada e 2,2 a 3,6 $\mathrm{m}^{3} / \mathrm{ha} /$ ano na área explorada com aplicação de tratamentos silviculturais.

De Graaf (1986), referindo-se a estudos de crescimento em floresta tropical natural no Suriname, observou aumento no incremento anual em volume de 2,0 a 4,5 $\mathrm{m}^{3} / \mathrm{ha}$ /ano nas espécies comerciais sob tratamentos silviculturais. Segundo Uhl et al. (1992), a adoção de técnicas silviculturais resulta em maior incremento anual em diâmetro. Segundo De Graff (1981), citado por Barreto et al. (1993), a taxa média de incremento diamétrico das árvores em florestas exploradas sem tratamentos silviculturais variou de 0,1 a 0,4 $\mathrm{cm} /$ ano, enquanto em florestas exploradas com tratamentos silviculturais, de 0,6 a $1,0 \mathrm{~cm} /$ ano.

\subsection{Custos e receitas}

Os custos envolvidos na exploração florestal podem ser divididos em custos de planejamento e de manejo. Em estudos conduzidos por Amaral et al. (1998), em áreas de floresta primária sob colheita de impacto reduzido na Amazônia, o custo total de exploração foi de US $\$ 30.3 / \mathrm{m}^{3}$ e a receita bruta, de US\$40.0/ $\mathrm{m}^{3}$. Em estudo semelhante, Barreto et al. (1998) encontraram um custo de US $\$ 26.48 / \mathrm{m}^{3}$ e uma receita bruta de US $\$ 40.80 / \mathrm{m}^{3}$. Em outro trabalho da mesma natureza, realizado por Holmes et al. (2002), o custo foi de US\$13.84/ $\mathrm{m}^{3}$ e a receita bruta, de US $\$ 25.50 / \mathrm{m}^{3}$.

Souza e Oliveira (1999), em estudo realizado no Centro de Pesquisa Florestal da GHETHAL, Itacoatiara, AM, evidenciou que o custo de exploração foi de US\$16.65/m³ . Van der Hout (1999), citado por Boltz et al. (2003), analisando uma floresta tropical natural sob colheita de impacto reduzido no Oeste da Guiana, constatou que o custo total de exploração foi de US\$16.05/ $/ \mathrm{m}^{3}$.

\section{MATERIAL E MÉTODOS}

O estudo foi realizado na unidade de manejo florestal (UMF) da Fazenda Tracajás (02³5'53'’S e 4747'10’'W), empresa Nova Era Agroflorestal, de propriedade do Grupo Rosa Madeireira, município de Paragominas, Estado do Pará, Brasil, cuja tipologia florestal foi classificada como floresta ombrófila densa de terra firme em estádio de sucessão primária (clímax).

Na execução do plano de manejo florestal, destinado à produção de madeira para serraria e laminação, foi realizado o inventário de prospecção (100\%), no qual se estimaram os volumes do fuste comercial das árvores com dap $\geq 45 \mathrm{~cm}$ de 55 espécies comerciais em 49 talhões de exploração de 10 ha cada um, perfazendo um total de 490 ha.

No inventário, $100 \%$ das árvores comerciais com dap $\geq 45 \mathrm{~cm}$ (diâmetro mínimo de corte permissível) foram identificadas, marcando-se as árvores-matriz, as árvores localizadas nas áreas de proteção ambiental e as selecionadas para corte.

A unidade de manejo florestal, isto é, os 49 talhões de exploração, foi estratificada em áreas homogêneas de florestas, com base no estoque de árvores comerciais das 55 espécies autorizadas para corte pelo IBAMA, doravante denominadas classes I, II e III de estoques volumétricos. Foram empregadas as técnicas de análises de agrupamento e discriminante na definição das respectivas classes de estoque.

$\mathrm{Na}$ análise de agrupamentos, os talhões ou unidades de trabalho formaram grupos homogêneos e distintos, agrupados nas classes I, II e III de estoques volumétricos, com os respectivos volumes mínimo, médio, máximo e desvios-padrão (Quadro 1).

Considerou-se como volume de corte autorizado pelo IBAMA as árvores comerciais com dap $\geq 45$ cm selecionadas no inventário de prospecção (100\%), ou seja, após descontados os volumes das árvoresmatriz e das árvores localizadas em áreas de proteção ambiental.

Em cada classe de estoque foram instaladas, aleatoriamente, cinco parcelas de 100 x $100 \mathrm{~m}$ ( 1 ha) cada uma, para a medição dos indivíduos com dap $\geq$ $15 \mathrm{~cm}$. No centro de cada parcela foi instalada uma subparcela de $10 \times 100 \mathrm{~m}(0,1$ ha), para a medição dos indivíduos com $5 \mathrm{~cm} \leq$ dap $<15 \mathrm{~cm}$.

$\mathrm{O}$ volume de fuste com casca de árvores individuais foi estimado pelo emprego da equação $\hat{Y}=0,0774759688+0,517896768\left(d a p^{2} \times H c\right)$, desenvolvida por Queiroz (1984).

R. Árvore, Viçosa-MG, v.28, n.5, p.681-689, 2004 
Quadro 1 - Caracterização das classes de estoque volumétrico, mediante os valores dos volumes mínimo, médio e máximo e dos desvios-padrão

Table 1 -Characterization of volumetric stock classes, value of standart deviation, volumes maximum, average and minimum

\begin{tabular}{lccrr}
\hline $\begin{array}{l}\text { Classe de } \\
\text { Estoque }\end{array}$ & Mínimo & \multicolumn{2}{c}{ Volume $\left(\mathrm{m}^{3} / \mathrm{ha}\right)$} \\
Médio & Máximo & $\begin{array}{r}\text { Desvio- } \\
\text { Padrão }\end{array}$ \\
\hline I & 35,60 & 45,16 & 50,89 & 4,67 \\
II & 52,55 & 58,11 & 63,82 & 3,41 \\
III & 69,50 & 76,77 & 89,36 & 6,37 \\
\hline
\end{tabular}

Os critérios de maximização da produção volumétrica do estoque total ou final $\left(V T_{j}\right)$, do estoque remanescente $\left(V R_{j}\right)$ e do estoque comercial $\left(V C_{j}\right)$ e o incremento médio anual $\left(\mathrm{Cr}_{j}\right)$, por classes I, II e III de estoques volumétricos, foram utilizados para simular alternativas de manejo, elaboradas por meio do emprego das expressões algébricas:

$$
\begin{gathered}
V T_{j}=V R_{j}\left(1+i_{j}\right)^{c c_{j}} \\
i_{j}=\frac{C r_{j}}{\left(\frac{V T_{j}+V R_{j}}{2}\right)} \\
c c_{j}=\frac{\ln \left(V T_{j}\right)-\ln \left(V R_{j}\right)}{\ln \left(1+i_{j}\right)} \\
V C_{j}=V T_{j}-V R_{j}
\end{gathered}
$$

em que $V T_{j}=$ volume do estoque total ou final na jésima alternativa de manejo $\left(\mathrm{m}^{3} / \mathrm{ha}\right), V R_{j}=$ volume de estoque remanescente na j-ésima alternativa de manejo $\left(\mathrm{m}^{3} / \mathrm{ha}\right), i_{j}=$ taxa de crescimento anual na j-ésima alternativa de manejo (\%), $c c_{j}=$ ciclo de corte na j-ésima alternativa de manejo (anos), $\mathrm{Cr}_{j}=$ incremento médio anual na j-ésima alternativa de manejo $\left(\mathrm{m}^{3 /}\right.$ ha/ano), $l n=$ logaritmo neperiano e $V C_{j}=$ volume de corte ou estoque comercial na j-ésima alternativa de manejo ( $\left.\mathrm{m}^{3} / \mathrm{ha}\right)$.

A projeção do estoque remanescente $(V R)$ fundamenta-se no pressuposto de que os crescimentos anuais de uma árvore se acumulam, seguindo a lei de juros compostos.

Foram geradas 12 alternativas de manejo $(\mathrm{j}=1$, $2, \ldots . .12$ ) em cada classe de estoque volumétrico, que resultaram da combinação de três níveis de colheita $\left(V C_{j}\right)$ e quatro valores de incremento médio anual em volume $\left(\mathrm{Cr}_{j}\right)$ (Quadro 2). A colheita baseou-se no diâmetro mínimo de corte permissível (dap $\geq 45 \mathrm{~cm}$ ) e no volume máximo de corte permissível (Vmáx $\left.=35 \mathrm{~m}^{3} / \mathrm{ha}\right)$ (Instrução Normativa/IBAMA, $\mathrm{N}^{\circ} 4$, de 2002) e no método de hectare eqüiprodutivo $\left(\mathrm{Heq}_{j}\right)$. Os valores do incremento médio anual $\left(\mathrm{Cr}_{j}\right)$ foram de 1,$5 ; 2,0 ; 2,5 ;$ e 3,0 $\mathrm{m}^{3} / \mathrm{ha} / \mathrm{ano}$.

O hectare eqüiprodutivo $\left(H e q_{j}\right)$ foi determinado com o emprego da seguinte expressão:

$$
\mathrm{Heq}_{j}=\left(\frac{\sum_{j=1}^{n} A_{j} \times V_{j}}{\sum_{j=1}^{n} A_{J}}\right) \div V_{j}
$$

em que $\mathrm{Heq}_{j}=$ hectare eqüiprodutivo na j-ésima classe de estoque, $A_{j}=$ área total da j-ésima classe de estoque, $V_{j}=$ volume $\mathrm{em} \mathrm{m}^{3} /$ ha do estoque comercial na j ésima classe de estoque e $\bar{V}_{j}=$ volume médio em $\mathrm{m}^{3} /$ ha ponderado do estoque comercial na j-ésima classe de estoque.

De posse do valor do hectare eqüiprodutivo na j-ésima classe de estoque $\left(\mathrm{Heq}_{\mathrm{j}}\right)$, calculou-se o volume de corte na j-ésima classe de estoque $\left(V C_{j}\right)$, com

\begin{tabular}{|c|c|c|c|c|c|c|c|c|c|c|c|c|}
\hline \multirow{2}{*}{$\begin{array}{l}\text { Nível de } \\
\text { Colheita }\end{array}$} & \multicolumn{4}{|c|}{ Classe I } & \multicolumn{4}{|c|}{ Classe II } & \multicolumn{4}{|c|}{ Classe III } \\
\hline & 1,5 & 2,0 & 2,5 & 3,0 & 1,5 & 2,0 & 2,5 & 3,0 & 1,5 & 2,0 & 2,5 & 3,0 \\
\hline dap $\geq 45 \mathrm{~cm}$ & 38 & 38 & 38 & 38 & 55 & 55 & 55 & 55 & 65 & 65 & 65 & 65 \\
\hline Vmáx & 35 & 35 & 35 & 35 & 35 & 35 & 35 & 35 & 35 & 35 & 35 & 35 \\
\hline Heq & 25 & 25 & 25 & 25 & 36 & 36 & 36 & 36 & 43 & 43 & 43 & 43 \\
\hline
\end{tabular}
o emprego da expressão:

$$
V C_{j}=\frac{V m a ́ x}{H e q_{j}}
$$

Quadro 2 - Alternativas de colheita de diferentes volumes e incrementos anuais em volume Table 2 -Alternative of the haverst for volumes diferent and increment annual in a volume 
O ciclo de corte econômico ótimo por classe de estoque volumétrico foi determinado como o nível em que a taxa anual do incremento periódico em valor do estoque comercial se iguala à taxa anual de juros (\%) oferecida pelo mercado de capital, com o emprego das expressões:

$$
\begin{gathered}
\Delta V M I P_{j t}(\%)=\left(\frac{V M I P_{j(t+1)}-V M I P_{j t}}{V M I P_{j t}}\right)-\left(\frac{C I P_{j(t+1)}-C I P_{j t}}{C I P_{j t}}\right) \times 100 \\
V M I P_{j(t+1)}=\left(V R_{j(t+1)}\left(1+i_{j}\right)^{t}-V R_{j t_{0}}\right) \times P_{j} \\
V M I P_{j t}=I P_{j t} \times P_{j} \\
C I P_{j(t+1)}=\left(V R_{j(t+1)}\left(1+i_{j}\right)^{t}-V R_{j t_{0}}\right) \times C_{j}
\end{gathered}
$$

em que $\Delta V M I P_{j t}(\%)=$ variação do valor monetário do incremento periódico do estoque comercial da j-ésima alternativa de manejo no período $t, V M I P_{\mathrm{j}(t+1)}=$ valor monetário do incremento periódico (US\$/ha) do estoque comercial da j-ésima alternativa de manejo no período $t+1, V M I P_{j t}=$ valor monetário do incremento periódico (US $\$ /$ ha) do estoque comercial da j-ésima alternativa de manejo no período $t, C I P_{j(t+1)}=$ custo do incremento periódico da j-ésima alternativa de manejo no período $t+1, C I P_{j t}=$ custo do incremento periódico da j-ésima alternativa de manejo no período $t$, $\mathrm{VR}_{j(t+l)}=$ volume remanescente $\left(\mathrm{m}^{3} / \mathrm{ha}\right) \mathrm{da}$ j-ésima alternativa de manejo no período $t+1, V R_{j t o}=$ volume remanescente $\left(\mathrm{m}^{3} / \mathrm{ha}\right)$ da j-ésima alternativa de manejo no período $t_{0}, P_{j}=$ preço da madeira em tora na j-ésima alternativa de manejo (US $\left.\$ / \mathrm{m}^{3}\right)$ e $C_{j}=$ custo da madeira em tora na j-ésima alternativa de manejo $\left(\mathrm{US} \$ / \mathrm{m}^{3}\right)$.

Para determinar o valor monetário líquido do incremento periódico (VMLIP) no período $t$ referente ao estoque comercial, consideraram-se dois cenários. No cenário 1, o preço e o custo da madeira em tora foram mantidos constantes. No cenário 2 , o preço variou a uma taxa de juros compostos de $1 \%$ ao ano e custo constante. O VMLIP foi calculado com o emprego da expressão:

$$
V M L I P_{j t}=V M I P_{j t}-C I P_{j t}
$$

em que $V M L I P_{j t}=$ valor monetário líquido do incremento periódico (US\$/ha) da j-ésima alternativa de manejo no período $t$.

O preço da madeira colhida posta no pátio da floresta e o custo médio de produção, independentemente da espécie, determinados por Holmes et al. (2002), foram de US $\$ 25.50 / \mathrm{m}^{3}$ e US $\$ 13.84 / \mathrm{m}^{3}$, respectivamente.

\section{RESULTADOS E DISCUSSÃO}

Para efeito de discussão dos resultados, considerou-se a taxa de juros oferecida pelo mercado de capital de no mínimo $6 \%$ ao ano e o corte permissível de no máximo $35 \mathrm{~m}^{3} / \mathrm{ha}$; e o ciclo de corte de no mínimo 25 anos (Instrução Normativa/IBAMA, N ${ }^{\circ}$, de maio de 2002).

Na classe I de estoque (Quadro 3), nos cenários 1 e 2 a intensidade de corte de $38 \mathrm{~m}^{3} /$ ha remunerou o manejo às taxas de 8,8 e 10,9\% no ciclo de corte de 13 anos, auferindo valores monetários líquidos do estoque comercial de US $\$ 453.00 /$ ha e US $\$ 589.81 / \mathrm{ha}$, respectivamente. $\mathrm{O}$ volume colhido de $35 \mathrm{~m}^{3} /$ ha remunerou o manejo às taxas de 9,5 e 11,7\% no ciclo de corte de 12 anos, com receitas líquidas do estoque comercial de US $\$ 418.23 /$ ha e US $\$ 534.23 /$ ha, respectivamente. A intensidade de corte de $25 \mathrm{~m}^{3} / \mathrm{h}$ a remunerou o manejo às taxas de 14,7 e 17,1\% no ciclo de corte de oito anos, com as respectivas receitas líquidas do estoque comercial de US\$278.21/ha e US\$382.62/ha. Dessa forma, pode-se inferir que os ciclos econômicos ótimos foram de 13, 12 e 8 anos, a um incremento médio anual de $3,0 \mathrm{~m}^{3} / \mathrm{ha} / \mathrm{ano}$, independentemente dos níveis de colheita, respectivamente (Quadro 3).

Na classe II de estoque (Quadro 4), nos cenários 1 e 2 a intensidade de corte de $55 \mathrm{~m}^{3} /$ ha remunerou o manejo às taxas de 6,4 e $8,3 \%$ no ciclo de corte de 18 anos, representando um valor monetário líquido do estoque comercial de US\$623.97/ha e US\$891.64/ ha, respectivamente. O volume colhido de $35 \mathrm{~m}^{3} / \mathrm{ha}$ remunerou o manejo às taxas de 9,6 e $11,7 \%$ no ciclo de corte de 12 anos, auferindo valores monetários líquidos do estoque comercial de US $\$ 418.05 /$ ha e US $\$ 534.00 /$ ha, respectivamente. A intensidade de corte de $36 \mathrm{~m}^{3 /}$ ha remunerou o manejo às taxas de 9,6 e $11,7 \%$ no ciclo de corte de 12 anos, representando valores monetários líquidos do estoque comercial de US $\$ 417.39 /$ ha e US\$533.16/ha, respectivamente. Portanto, pode-se inferir que os ciclos econômicos ótimos foram de 18 , 12 e 12 anos, a um incremento médio anual de $3,0 \mathrm{~m}^{3} /$ ha/ano nos diferentes níveis de colheita, respectivamente (Quadro 4).

Na classe III de estoque (Quadro 5), nos cenários 1 e 2 a intensidade de corte de $65 \mathrm{~m}^{3} / \mathrm{ha} \mathrm{remu-}$ nerou o manejo às taxas de 5,3 e 7,2\% no ciclo de corte de 22 anos, auferindo valores monetários líquidos do estoque comercial de US\$763.65/ha e US $\$ 1172.29 / \mathrm{ha}$,

R. Árvore, Viçosa-MG, v.28, n.5, p.681-689, 2004 
respectivamente. No cenário 1 , a taxa de 5,3\% encontrase abaixo do mínimo oferecido pelo mercado, que é de $6 \%$ ao ano. O volume colhido de $35 \mathrm{~m}^{3} /$ ha remunerou o manejo às taxas de 9,6 e 11,7\% no ciclo de corte de 12 anos, representando valores monetários líquidos do estoque comercial de US\$417.96/ha e US $\$ 533.88 /$ ha, respectivamente. A intensidade de corte de $43 \mathrm{~m}^{3} /$ ha remunerou o manejo às taxas de 8,2 e $10,3 \%$ no ciclo de corte de 14 anos, auferindo valores monetários líquidos do estoque comercial de US\$485.74/ha e US\$644.53/ha, respectivamente. Dessa forma, podese inferir que os ciclos econômicos ótimos foram de 22 (cenário 2), 12 e 14 anos, a um incremento médio anual de $3,0 \mathrm{~m}^{3} / \mathrm{ha} /$ ano nas diferentes intensidades de corte, respectivamente (Quadro 5).

Nas classes I, II e III de estoque, verificou-se que maiores taxas de crescimento resultaram em menores ciclos de corte, independentemente dos níveis de colheita do estoque comercial. Maiores incrementos anuais em volume resultaram também em maiores taxas de valoração da floresta. No entanto, maiores volumes colhidos implicaram ciclos de corte mais longos numa mesma taxa de crescimento. Contudo, dentro de certos limites, maiores volumes colhidos podem resultar biologicamente em maiores taxas de crescimento do estoque remanescente. Isso ocorre, principalmente, quando se aplicam tratamentos silviculturais.

De acordo Souza (1999), ciclos de cortes mais curtos e frequientes são aconselháveis para melhorar a composição florística e a distribuição diamétrica desejável e manter a produção sustentável. Além do mais, um ciclo de corte curto permite maior controle biológico do povoamento, maximiza o crescimento de madeira, melhora a qualidade da madeira e mantém as condições multiâneas da floresta. Contudo, a intensidade de colheita deve ser mantida no nível mais baixo possível, para não comprometer a sustentabilidade do recurso.

Analisando o cenário 1, nas classes I, II e III de estoques volumétricos, verificou-se que nenhuma das alternativas de manejo atende simultaneamente às restrições estabelecidas pelo IBAMA (volume de corte de $35 \mathrm{~m}^{3} /$ ha e ciclo de corte de 25 anos) e taxa de crescimento do incremento de no mínimo $6 \%$ ao ano.

No cenário 1, nas classes I, II e III de estoques volumétricos o ciclo de corte que atende à taxa de crescimento do incremento de no mínimo $6 \%$ ao ano deveria ser de no máximo 18 anos, à taxa de 2,0 $\mathrm{m}^{3} /$ ha/ano, enquanto no cenário 2 o ciclo de corte deveria ser de no máximo 23 anos, a mais baixa taxa de crescimento $\left(1,5 \mathrm{~m}^{3} / \mathrm{ha} / \mathrm{ano}\right)$.

Quadro 3 - Volume total $(V T)$, volume remanescente $(V R)$ e volume de corte $(V C)$, incremento anual $(C r)$, ciclo de corte $(c c)$, variação do valor monetário do incremento periódico $(\triangle V M I P)$ e valor monetário líquido do incremento periódico $(V M L I P)$, na classe I de estoque. Fazenda Tracajás, município de Paragominas, Estado do Pará

Table 3 - Total volume (VT), remaining volume (VR), cut volume (VC), annual increment (Cr), cut cycle (cc), variation of monetary value of periodic increment $(\triangle V M I P)$ ) and net profit monetary value of periodic increment $(V M L I P)$, stock class I, Tracajás Farm, municipality Paragominas, Pará state

\begin{tabular}{|c|c|c|c|c|c|c|c|c|c|}
\hline \multirow{2}{*}{$\begin{array}{l}\text { Alternativa } \\
\text { de Manejo }\end{array}$} & \multicolumn{3}{|c|}{ Volume $\left(\mathrm{m}^{3} / \mathrm{ha}\right)$} & \multirow{2}{*}{$\begin{array}{c}\mathrm{Cr} \\
\mathrm{m}^{3} / \mathrm{ha} / \mathrm{ano} \\
\end{array}$} & \multirow{2}{*}{$\begin{array}{c}\mathrm{cc} \\
\text { Anos } \\
\end{array}$} & \multicolumn{2}{|c|}{ CENÁRIO 1} & \multicolumn{2}{|c|}{ CENÁRIO 2} \\
\hline & VT & VR & $\mathrm{VC}$ & & & $\begin{array}{c}\Delta \mathrm{VMIP} \\
\%\end{array}$ & $\begin{array}{l}\text { VMLIP } \\
\text { US\$/ha }\end{array}$ & $\begin{array}{c}\Delta \mathrm{VMIP} \\
\% \\
\end{array}$ & $\begin{array}{l}\text { VMLIP } \\
\text { US\$/ha }\end{array}$ \\
\hline 1 & 387,61 & 349,61 & 38,00 & 1,50 & 25 & 4,4 & 435.61 & 6,2 & 704.67 \\
\hline 2 & 387,61 & 349,61 & 38,00 & 2,00 & 19 & 5,8 & 441.41 & 7,8 & 642.30 \\
\hline 3 & 387,61 & 349,61 & 38,00 & 2,50 & 15 & 7,5 & 434.99 & 9,5 & 588.12 \\
\hline 4 & 387,61 & 349,61 & 38,00 & 3,00 & 13 & 8,8 & 453.00 & 10,9 & 589.81 \\
\hline 5 & 387,61 & 352,61 & 35,00 & 1,50 & 23 & 4,8 & 400.83 & 6,6 & 626.26 \\
\hline 6 & 387,61 & 352,61 & 35,00 & 2,00 & 18 & 6,2 & 418.82 & 8,1 & 598.48 \\
\hline 7 & 387,61 & 352,61 & 35,00 & 2,50 & 14 & 8,1 & 406.34 & 10,1 & 539.18 \\
\hline 8 & 387,61 & 352,61 & 35,00 & 3,00 & 12 & 9,5 & 418.23 & 11,7 & 534.23 \\
\hline 9 & 387,61 & 362,61 & 25,00 & 1,50 & 17 & 6,5 & 296.80 & 8,4 & 416.43 \\
\hline 10 & 387,61 & 362,61 & 25,00 & 2,00 & 13 & 8,6 & 302.62 & 10,7 & 394.01 \\
\hline 11 & 387,61 & 362,61 & 25,00 & 2,50 & 10 & 11,5 & 290.39 & 13,7 & 356.83 \\
\hline 12 & 387,61 & 362,61 & 25,00 & 3,00 & 8 & 14,7 & 278.21 & 17,1 & 328.62 \\
\hline
\end{tabular}

R. Árvore, Viçosa-MG, v.28, n.5, p.681-689, 2004 
Quadro 4 - Volume total $(V T)$, volume remanescente $(V R)$ e volume de corte $(V C)$, incremento anual $(C r)$, ciclo de corte $(c c)$, variação do valor monetário do incremento periódico $(\Delta V M I P)$ e valor monetário líquido do incremento periódico $(V M L I P)$, na classe I de estoque. Fazenda Tracajás, município de Paragominas, Estado do Pará

Table 4 - Total volume (VT), remaining volume (VR), cut volume (VC), annual increment (Cr), cut cycle (cc), variation of monetary value of periodic increment $(\triangle V M I P)$ ) and net profit monetary value of periodic increment (VMLIP), stock class II, Tracajás Farm, municipality Paragominas, Pará state

\begin{tabular}{|c|c|c|c|c|c|c|c|c|c|}
\hline \multirow{2}{*}{$\begin{array}{l}\text { Alternativa } \\
\text { de Manejo }\end{array}$} & \multicolumn{3}{|c|}{ Volume $\left(\mathrm{m}^{3} / \mathrm{ha}\right)$} & \multirow{2}{*}{$\begin{array}{c}\mathrm{Cr} \\
\mathrm{m}^{3} / \mathrm{ha} / \text { ano }\end{array}$} & \multirow{2}{*}{$\begin{array}{c}\text { cc } \\
\text { Anos }\end{array}$} & \multicolumn{2}{|c|}{ CENÁRIO 1} & \multicolumn{2}{|c|}{ CENÁRIO 2} \\
\hline & VT & VR & $\mathrm{VC}$ & & & $\underset{\%}{\Delta \text { VMIP }}$ & $\begin{array}{l}\text { VMLIP } \\
\text { US\$/ha }\end{array}$ & $\begin{array}{c}\text { DVMIP } \\
\%\end{array}$ & $\begin{array}{l}\text { VMLIP } \\
\text { US\$/ha }\end{array}$ \\
\hline 1 & 355,98 & 300,98 & 55,00 & 1,50 & 37 & 3,0 & 644.39 & 4,7 & 1271.62 \\
\hline 2 & 355,98 & 300,98 & 55,00 & 2,00 & 28 & 4,0 & 650.17 & 5,8 & 1107.01 \\
\hline 3 & 355,98 & 300,98 & 55,00 & 2,50 & 22 & 5,2 & 637.04 & 7,1 & 977.97 \\
\hline 4 & 355,98 & 300,98 & 55,00 & 3,00 & 18 & 6,4 & 623.97 & 8,3 & 891.64 \\
\hline 5 & 355,98 & 320,98 & 35,00 & 1,50 & 23 & 4,8 & 400.66 & 6,6 & 625.99 \\
\hline 6 & 355,98 & 320,98 & 35,00 & 2,00 & 18 & 6,2 & 418.69 & 8,2 & 598.30 \\
\hline 7 & 355,98 & 320,98 & 35,00 & 2,50 & 14 & 8,1 & 406.14 & 10,2 & 538.91 \\
\hline 8 & 355,98 & 320,98 & 35,00 & 3,00 & 12 & 9,6 & 418.05 & 11,7 & 534.00 \\
\hline 9 & 355,98 & 319,98 & 36,00 & 1,50 & 24 & 4,6 & 418.36 & 6,4 & 665.16 \\
\hline 10 & 355,98 & 319,98 & 36,00 & 2,00 & 18 & 6,2 & 418.04 & 8,2 & 597.36 \\
\hline 11 & 355,98 & 319,98 & 36,00 & 2,50 & 14 & 8,1 & 405.50 & 10,2 & 538.06 \\
\hline 12 & 355,98 & 319,98 & 36,00 & 3,00 & 12 & 9,6 & 417.39 & 11,7 & 533.16 \\
\hline
\end{tabular}

Quadro 5 - Volume total $(V T)$, volume remanescente $(V R)$ e volume de corte $(V C)$, incremento anual $(C r)$, ciclo de corte $(c c)$, variação do valor monetário do incremento periódico (DVMIP) e valor monetário líquido do incremento periódico $(V M L I P)$, na classe I de estoque. Fazenda Tracajás, município de Paragominas, Estado do Pará

Table 3 - Total volume (VT), remaining volume (VR), cut volume (VC), annual increment (Cr), cut cycle (cc), variation of monetary value of periodic increment (DVMIP)) and net profit monetary value of periodic increment (VMLIP), stock class III, Tracajás Farm, municipality Paragominas, Pará state

\begin{tabular}{|c|c|c|c|c|c|c|c|c|c|}
\hline \multirow{2}{*}{$\begin{array}{l}\text { Alternativa } \\
\text { de Manejo }\end{array}$} & \multicolumn{3}{|c|}{ Volume $\left(\mathrm{m}^{3} / \mathrm{ha}\right)$} & \multirow{2}{*}{$\begin{array}{c}\mathrm{Cr} \\
\mathrm{m}^{3} / \mathrm{ha} / \text { ano }\end{array}$} & \multirow{2}{*}{$\begin{array}{l}\mathrm{cc} \\
\text { Anos }\end{array}$} & \multicolumn{2}{|c|}{ CENÁRIO 1} & \multicolumn{2}{|c|}{ CENÁRIO 2} \\
\hline & VT & VR & $\mathrm{VC}$ & & & $\begin{array}{c}\Delta \mathrm{VMIP} \\
\% \\
\end{array}$ & $\begin{array}{l}\text { VMLIP } \\
\text { US\$/ha }\end{array}$ & $\begin{array}{c}\text { DVMIP } \\
\% \\
\end{array}$ & $\begin{array}{l}\text { VMLIP } \\
\text { US\$/ha }\end{array}$ \\
\hline 1 & 342,64 & 277,64 & 65,00 & 1,50 & 44 & 2,6 & 765.65 & 4,2 & 1685.46 \\
\hline 2 & 342,64 & 277,64 & 65,00 & 2,00 & 33 & 3,5 & 764.94 & 5,2 & 1415.24 \\
\hline 3 & 342,64 & 277,64 & 65,00 & 2,50 & 26 & 4,4 & 751.46 & 6,2 & 1236.69 \\
\hline 4 & 342,64 & 277,64 & 65,00 & 3,00 & 22 & 5,3 & 763.61 & 7,2 & 1172.29 \\
\hline 5 & 342,64 & 307,64 & 35,00 & 1,50 & 23 & 4,8 & 400.57 & 6,6 & 625.86 \\
\hline 6 & 342,64 & 307,64 & 35,00 & 2,00 & 18 & 6,2 & 418.63 & 8,2 & 598.21 \\
\hline 7 & 342,64 & 307,64 & 35,00 & 2,50 & 14 & 8,1 & 406.04 & 10,2 & 538.77 \\
\hline 8 & 342,64 & 307,64 & 35,00 & 3,00 & 12 & 9,6 & 417.96 & 11,7 & 533.88 \\
\hline 9 & 342,64 & 299,64 & 43,00 & 1,50 & 29 & 3,8 & 505.54 & 5,6 & 875.37 \\
\hline 10 & 342,64 & 299,64 & 43,00 & 2,00 & 22 & 5,1 & 511.33 & 7,0 & 784.99 \\
\hline 11 & 342,64 & 299,64 & 43,00 & 2,50 & 17 & 6,7 & 492.32 & 8,7 & 690.76 \\
\hline 12 & 342,64 & 299,64 & 43,00 & 3,00 & 14 & 8,2 & 485.74 & 10,3 & 644.53 \\
\hline
\end{tabular}

Em termos comparativos, prefixando o ciclo de corte de 25 anos e o volume de corte de $35 \mathrm{~m}^{3} /$ ha (Instrução Normativa/IBAMA, $\mathrm{N}^{\circ} 4$, maio de 2002), no cenário 1 a intensidade de corte que propiciaria uma taxa de retorno compatível com a taxa de juros de mercado (6\% a.a.) deveria ser de $25 \mathrm{~m}^{3} / \mathrm{ha}$ e o ciclo de corte, de no máximo 18 anos; enquanto no cenário 2 a intensidade de corte deveria ser de $30,62 \mathrm{~m}^{3} / \mathrm{ha}$ e o ciclo de corte de, no máximo, 23 anos.

Do ponto de vista de sustentação dos níveis de colheita apresentados, verificou-se que os estoques de colheita ótimos nas classes I, II e III de estoques 
volumétricos foram de $25 \mathrm{~m}^{3} / \mathrm{ha}, 36 \mathrm{~m}^{3} / \mathrm{ha} \mathrm{e} 35 \mathrm{~m}^{3} / \mathrm{ha}$, nos ciclos de corte de 17 e 18 anos, respectivamente. Com essas taxas de colheitas, é possível utilizar o recurso sem comprometer a sua capacidade de renovação ou sustentação da floresta manejada; sobretudo o manejo florestal conduzido a taxas de juros equivalentes à taxa de crescimento da floresta garante a produção sustentável de madeira e os retornos de investimento do capital na atividade de manejo.

Segundo Angelo e Guimarães (2001), o baixo preço da madeira em tora, o longo ciclo de corte, a baixa produtividade da floresta e a carência de crédito a juros compatíveis com taxa de crescimento da floresta são as principais limitações para a atividade de manejo.

Vale ressaltar, ainda, que o acréscimo anual no preço da madeira (cenário 2) contribuiu para a maior taxa de remuneração e retorno do investimento do capital no manejo.

\section{CONCLUSÕES}

- Menores incrementos e maiores intensidades de corte resultam também em maiores ciclos de corte e menores retornos financeiros da atividade de manejo.

- A atividade de manejo é viável economicamente a taxas de crescimento mais altas e a baixas taxas de juros do mercado.

- A taxa de crescimento da floresta manejada deve ser pelo menos igual ao custo de oportunidade do capital investido no manejo, para que a atividade seja uma alternativa economicamente atrativa.

- Pequeno acréscimo anual no preço da madeira indica que a atividade de manejo é rentável mesmo nos maiores ciclos de corte e baixas taxas de crescimento da floresta.

- A análise econômica mediante a combinação das taxas de investimento e crescimento da floresta aponta a necessidade de mais estudos na escolha do ciclo de corte e nível de colheita de cada floresta.

- A aplicação de sistema silvicultural policíclico logo após a exploração prioriza as espécies comerciais e potencialmente comercias, resultando em maiores taxas de crescimento e rentabilidade do manejo.

\section{REFERÊNCIAS BIBLIOGRÁFICAS}

ALDER, D.; SILVA, J. N. M. An empirical model for management of Terra Firme forests in the Brazilian Amazon. Forest Ecology and Management, v. 130, p. 141-157, 2000.

AMARAL, P. et al. Floresta para sempre: um manual para a produção de madeira na Amazônia. Belém: 1998. 155 p.

ANGELO, H.; GUIMARÃES, D. P. Subsídios econômicos ao manejo florestal sustentável na região amazônica. Revista Árvore, v. 25, n. 3, p. $353-360,2001$

ARAÚJO, P. A. et al. Idade relativa como subsídio à determinação de ciclo de corte no manejo sustentável de povoamentos florestais nativos. Revista Árvore, v. 17, n. 1, p. 100-106, 1993.

BARRETO, G. P.; UHL, C.; YARED, J. A.G. O potencial de produção sustentável de madeira em Paragominas - Pará; Amazônia Oriental: considerações ecológicas e econômicas. In: CONGRESSO FLORESTAL PANAMERICANO, 1 ; CONGRESSO FLORESTAL BRASILEIRO, 7., 1993, Curitiba. Anais... Curitiba: 1993. p.387-392.

BARRETO, P. et al. Costs and benefits of forest management for timber production in eastern Amazonia. Forest Ecology and Management, v. 108, p. 9-26, 1998.

BOM, R. P. Proposição de um sistema de manejo para floresta nativa objetivando a sustentabilidade da produção. 1996. $199 \mathrm{f}$. Tese (Doutorado em Ciência Florestal) - Universidade Federal do Paraná, Curitiba, 1996.

BOLTZ, F.; HOLMES, T. P.; CARTER, D. R. Economic and enviromental impacts of convencional and reduced-impact logging in tropical South America: a comparative review. Forest Policy and Economics, v. 5, p. 69$81,2003$.

CHICHORRO, J. F. Análise estrutural e econômica de multiprodutos da madeira em florestas naturais. 2000. 241 f. Tese (Doutorado em Ciência Florestal) Universidade Federal de Viçosa, Viçosa, 2000. 
De GRAAF, N. R. Silvicultural system for natural regeneration of tropical rain forest in Suriname. Wageningen: Agricultural University, 1986. 250 p.

FERREIRA, R. L. C. Estrutura e dinâmica de uma floresta secundária de transição, Rio Vermelho e Serra Azul de Minas, MG. 1997. $208 \mathrm{f}$. Tese (Doutorado em Ciência Florestal) - Universidade Federal de Viçosa, Viçosa, 1997.

HOLMES, T. P. et al. Financial and ecological indicators of reduced impact logging performance in the eastern amazon. Forest Ecology and Management, v. 163, p. 93-110, 2002.

QUEIROZ, W. T. Análise de fatores ("Factor Analysis") pelo método da máxima verossimilhança: aplicação ao estudo da estrutura de florestas tropicais. 1984. $109 \mathrm{f}$. Tese (Doutorado em Engenharia Florestal) - Universidade Federal do Paraná, Curitiba, 1984.

MAITRE, H. F. Silvicultural interventions and their effects on forest dynamics and production in some rain forests of cote d'ivoire. In: POMPAGÓMEZ, A.; WHITMORE, T. C.; HADLEY, M.

Rain forest regeneration and management. Paris: UNESCOP, 1991. v.6. p.383-392.
SILVA, J. N. M.; CARVALHO, J. O. P.; LOPES, J. C. A. Um sistema silvicultural policíclico para produção sustentada de madeira na amazônia brasileira. In: SIMPÓSIO SILVICULTURAL NA AMAZÔNIA ORIENTAL., 1999, Belém. Resumos expandidos.... Belém: EMBRAPACPATU/DFID, 1999. p. 180-185. (EMBRAPACPATU. Documentos, 123).

SILVA, J. N. M. et al. Growth and yeld of a tropical rain forest in the Brazilian Amazon 13 years after logging. Forest Ecology and Management, v. 71, p. 267-274, 1995.

SILVA, J. N. M. et al. Growth and yeld studies in the Tapajós region, Central Brazilian.

Commonwealth Forestry Review, v. 75, n. 4, p. 325-329, 1996.

SILVA, L. A . Ciclo de corte em florestas naturais secundárias nos municípios de Rio Vermelho e Serra Azul de Minas, Minas Gerais. 1996. $73 \mathrm{f}$. Dissertação (Mestrado em Ciência Florestal) Universidade Federal de Viçosa, Viçosa, 1996.

SOUZA, A. L. Estrutura, dinâmica e manejo de florestas tropicais. Viçosa: UFV, 1999. 122 p. (Apostila).

SOUZA, D. R.; OLIVEIA, S. A. Avaliação de custos das operações de exploração florestal em terra firme - Projeto Santa Rita, GETHAL Itacoatiara. In: SIMPÓSIO INTERNACIONAL SOBRE ECOSSISTEMAS FLORESTAIS, 5., 1999, Curitiba. Anais... Curitiba: 1999. Bio 1156.

UHL, C. et al. A evolução da fronteira amazônica - oportunidades para um desenvolvimento sustentável. In: PARÁ Desenvolvimento. Belém: IDESP, 1992. p. 13-21. 